\title{
Application of Multi Objective Genetic Algorithm with Pareto Optimal Solution Space for Design in IoT
}

\author{
Manas Kumar Yogi ${ }^{1}$, Yamuna Lakkamsani ${ }^{2}$ \\ Assistant Professor, Computer Science and Engineering, Pragati Engineering College, Kakinada, India ${ }^{1,2}$
}

\begin{abstract}
In this paper we consider the design issues in IoT and find that single heuristics cannot be used to optimize multiple objectives .So, we prefer a multi objective genetic algorithm approach to solve this design issues. We particularly consider the Pareto optimal solution which cannot be dominated by any other solution in solution space. We formulate 4 objectives for IoT design and try to minimize or maximize them as per the need in hand. The main aim is to obtain a refined IoT architecture. In a refined IoT architecture, design parameters are same but the input values to this parameters are within a boundary as specified by the Pareto solution set. This paper will help the IoT designer to consider the objective function in terms of IoT design factor and obtain a justifiable solution set to enhance the design ability of the IoT architecture.
\end{abstract}

Keywords: IoT, Genetic Algorithm, Pareto Optimal Solution, Multiobjective Optimization.

\section{INTRODUCTION}

In design of IOT the distributed functionality forces the designer to consider multiple factors like node capacity, co-operate cache coherence fault-tolerance security, privacy, scalability .these apart from the environmental heterogeneity makes the task more challenging for IOT designer so, we regard the design of IOT as multi objective optimization problem using genetic algorithm for multi objective problems the objective are conflicting in nature preventing optimization of each objective at same time it means when cost is minimized, performance is maximized and reliability is maximized ,security may be reduced and vice-versa. These are difficult but we need to really address them. Genetic algorithms are best suited given their multi-objective optimization heuristics to solve such problems.the idea we propose deals with modified $\mathrm{GA}$ for meeting the IoT design factors.

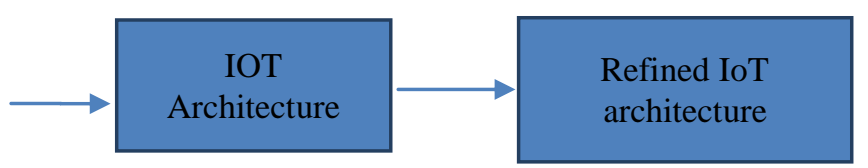

Fig 1: Design of the process.

In GA theory 2 methods of optimization are used

1. Add all individual objective into single objective function.

2. Separate all but one objective to the constraint-set.

whatever approach is followed even a person with deep domain knowledge will find it difficult due to fact that in first approach determining single objective is possible with utility theory weighted sum method but selection of proper weights depends on decision makers preference using latter approach ,moving all but one objective to constraint set needs a suitable constraining-value to be established for each of the objectives which is more tough than said in both cases tradeoffs exit ,so preferring a set of solution will be more conductive to the solution to the optimization problem.

The second approach we formulate in this paper refers to pre optimization solution set its set of solutions which are non-conflicting to each other of course selecting a Pareto solution over other needs some sacrifices in one objective in return for some gain.

\section{FORMULATION OF MULTI-OBJECTIVE OPTIMIZATION}

Consider an IoT design engineer whose need is to optimize k objectives which are non-conflicting in nature additionally, the decision maker has no priority of the objectives relative to each other. We have 2 types of objective functions minimization and maximization type. A minimization type by multiplying negative one.

Let us enumerate the objective which have to be either maximized or minimized for IoT design

Objective 1: Battery consumption at node should be minimized.

Objective 2: Privacy security at node should be maximized.

Objective 3: scalability should be maximized.

Objective 4: Heterogeneity should be maximized.

if we represents the above mentioned objectives as a set of $\mathrm{k}$ objectives functions in a $\mathrm{n}$-dimensional decision variable vector $x=\left\{x_{1}, x_{2} \ldots \ldots \ldots x_{n}\right\}$ in the solution space $X$, we have to determine the vector $x^{*}$ which maximizes the $\mathrm{k}$ objective function such that following equation holds true. 


$$
\mathrm{Z}\left(\mathrm{x}^{*}\right)=\left\{\mathrm{z}_{1}\left(\mathrm{x}^{*}\right), \mathrm{Z}_{2}\left(\mathrm{x}^{*}\right), \ldots . \mathrm{Z}_{\mathrm{k}}\left(\mathrm{x}^{*}\right)\right\}
$$

The solution space $\mathrm{X}$ is limited due to the series of constraints like $g_{j}\left(x^{*}\right)=b_{j}$ for $j=1,2,3 \ldots \ldots m b=$ bound variable.

While IoT design, considering given objectives, may conflict in real life situations a justifiable solution for multi objective problem is to investigate a set of solution, each of which satisfies the objectives at an acceptable level without being over shadowed by any other solution .For example, the battery life at a node in IoT cannot be minimized at the cost of enhanced security, privacy.

In our discussion we consider a Pareto optimal solution .It is defined as a solution which is not dominated by any other solution in the solution space. A feature of Pareto optimal solution is that it cannot be improved with respect to any objective without worsening at least one other objective. Set of all feasible non-dominated solutions is called Pareto optimal set. The corresponding objective function values in objective space are called the Pareto front the goal of the IoT designer is to find the best known solution from the Pareto optimal set.

In equation 1 stated above $\mathrm{z}_{1}\left(\mathrm{x}^{*}\right)$ represents solution to objective function $1, \mathrm{z}_{2}\left(\mathrm{x}^{*}\right)$ represents solution to objective function and so on till solution to all desired objective function are met .

The Pareto front of each objective function is diverse in nature which is discussed in next section.

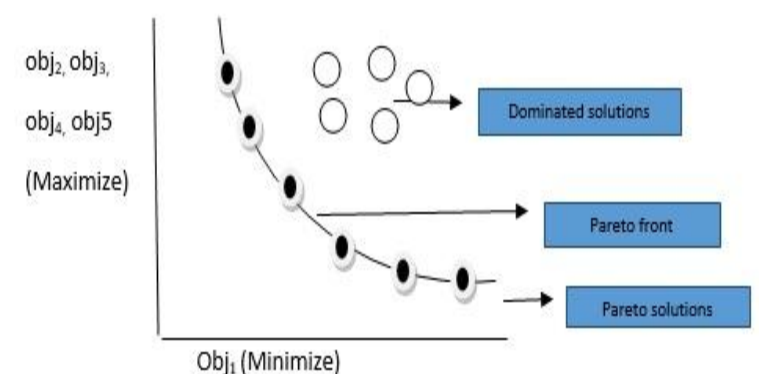

Fig 2: Below shows application of Pareto optimality in IoT design.

In IoT design

For objective1 i.e battery life has to be maximized which mean energy consumption to be minimized which depends on factor like component selection communication protocol application.

$\mathrm{Z}_{1}\left(\mathrm{x}^{*}\right)$ needs to be minimized in such a way that it should not cross the boundary set by the IoT designer the boundary values of input variables depends on factors which decrease energy consumption of a battery of a node. The mathematical expression is formatted as,

$\mathrm{g}_{\mathrm{j}}\left(\mathrm{x}_{1} *\right)=\mathrm{bj}_{1}\left(\mathrm{obj}_{1}\right)+\mathrm{bj}_{2}\left(\mathrm{obj}_{1}\right)+\mathrm{bj}_{3}\left(\mathrm{obj}_{1}\right)+\ldots \ldots . . \mathrm{bj}_{\mathrm{n}}\left(\mathrm{obj}_{1}\right)$

$\mathrm{J}_{1}=$ component selection

$\mathrm{J}_{2}=$ communication protocol

$\mathrm{J}_{3}=$ application

For objective 2, privacy security to be maximized $z\left(x^{*}\right)$ has to be maximized for that we need to consider which security measures are used high security measures may be provided by encryption algorithm but the encryption algorithm should be bounded by the size of the key for encryption decryption as larger size keys are time consuming security at the cost of operation time is a tradeoff.

The mathematical expression can be formulated as $\mathrm{g}_{\mathrm{j}}\left(\mathrm{x}_{2}{ }^{*}\right)=\mathrm{bj}_{1}\left(\mathrm{obj}_{2}\right)+\mathrm{bj}_{2}\left(\mathrm{obj} \mathrm{j}_{2}\right)+\ldots \ldots . .+\mathrm{bj}_{\mathrm{n}}\left(\mathrm{obj}_{2}\right)$

Where $J_{1}=$ Encryption algorithm with key size $n_{1}$.

$\mathrm{J}_{2}=$ Encryption algorithm with key size $\mathrm{n}_{2}$

$\mathrm{J}_{3}=$ Encryption algorithm with key size $n_{3}$.

For objective 3 i.e., scalability maximization constraint fixation depends on future need in IoT environmental with time scalable nodes need interaction to do this an proper device scale plan should be in order maintenance cost should be made a factor while taking final decision on the scaling issue .so, in this case the mathematical expression is, $g_{j}\left(x_{3} *\right)=b j_{1}\left(o b j_{3}\right)+b j_{2}\left(o b j_{3}\right)+\ldots \ldots \ldots \ldots \ldots \ldots \ldots+b j_{n}\left(o b j_{3}\right)$

Where $\mathrm{j}_{1}, \mathrm{j}_{2}, \mathrm{j}_{3}$ indicates maintenance cost after scaled IoT environmental is functional.

For objective 4, heterogeneity maximization the degree of interoperability in IoT should be increased in IoT should be increased. Intelligent agents are used in middle ware architecture of IoT which synchronize with corresponding agents and provide IoT functionalities .so, in this case the constraints will be the cost of deploying a specific software agent's .The mathematical expression is, $\mathrm{g}_{\mathrm{j}}\left(\mathrm{x}_{4} *\right)=\mathrm{bj}_{1}\left(\mathrm{obj}_{4}\right)+\mathrm{bj}_{2}\left(\mathrm{obj}_{4}\right)+\ldots \ldots \ldots \ldots \ldots \ldots \ldots . .+\mathrm{bj}_{\mathrm{n}}\left(\mathrm{obj}_{4}\right)$ $\mathrm{j}_{1}, \mathrm{j}_{2}, \mathrm{j}_{\mathrm{n}}$ represent cost of deploying $1,2, \ldots \mathrm{n}$ software agents in IoT network.

For objective 5 , fault tolerance to be maximized we take into account the downtime and update time of a node which fails during operation the down time should be minimized to do that nodes should be robust and have proper techniques which should be quickly activated during failure .so we, model below expression for maximizing objectives 5 .

$\mathrm{g}_{\mathrm{j}}\left(\mathrm{x}_{5} *\right)=\mathrm{bj}_{1}\left(\mathrm{obj}_{5}\right)+\mathrm{bj}_{2}\left(\mathrm{obj}_{5}\right)+\ldots \ldots . .+\mathrm{bj}_{\mathrm{n}}\left(\mathrm{obj}_{5}\right)$

Where $\mathrm{j}_{1}, \mathrm{j}_{2}, \mathrm{j}_{\mathrm{n}}$ indicate the tight downtime form nodes.

Hence $\mathrm{g}_{\mathrm{j}}\left(\mathrm{x}_{1} *\right), \mathrm{g}\left(\mathrm{x}_{2} *\right), \mathrm{g}_{\mathrm{j}}\left(\mathrm{x}_{3} *\right), \mathrm{g}\left(\mathrm{x}_{4} *\right), \mathrm{g}_{\mathrm{j}}\left(\mathrm{x}_{5}^{*}\right)$

Using $g_{j}\left(x^{*}\right)$ if we get proper optimal solution then the IoT designer prefers it over other feasible solution In case the designer wants to select another solution from the optimal solution set,it cannot be done without modification in values of any of theg $\mathrm{g}_{\mathrm{j}}\left(\mathrm{x}_{1}{ }^{*}, \mathrm{x}_{2} * \ldots . \mathrm{x}_{5} *\right)$ values subsequently we can assure that an efficient multi-objective optimization mechanism for IoT design has been established.

\section{CONCLUSION}

We have presented few novel directions to leverage the design process of IoT architecture by putting forth a mechanism which presents application of Pareto optimal solution set concept. In this paper, we have considered multiobjective optimization problem to solve IoT design 
issues which are in turn objective functions which must be either minimized or maximized. This paper will act as readymade guide for IoT designers who need to think clearly what factors in IoT design affect the objectives of high quality IoT design. Subsequently we conclude that Genetic algorithms are efficient for application to IoT design due to their ability to handle objective functions with conflicting interests. The Pareto optimal solution space aggressively tries to solve this emerging issue in field of IoT design.

An easy way to comply with the conference paper formatting requirements is to use this document as a template and simply type your text into it.

\section{REFERENCES}

[1] S. M. Metev and V. P. Veiko, Laser Assisted Micro technology, 2nd ed., R. M. Osgood, Jr., Ed. Berlin, Germany: Springer-Verlag, 1998.

[2] Multi-objective optimization using genetic algorithms: A tutorial Abdullah Konaka,_, David W. Coitb, Alice E. Smith, ELSEVIER Reliability Engineering and System Safety 91 (2006) 992-1007.

[3] L. Atzori, A. Iera, and G. Morabito, "The Internet of Things: a survey," Computer networks, vol. 54, no. 15, pp. 2787-2805, 2010

[4] Deb K. Multi-objective optimization using evolutionary algorithms. New York: Wiley; 2001.

[5] Martorell S, Villanueva JF, Carlos S, Nebot Y, Sanchez A, Pitarch $\mathrm{JL}$, et al. RAMS+C informed decision-making with application to multi-objective optimization of technical specifications and maintenance using genetic algorithms. Reliab Eng Syst Safety 2005; 87(1):65-75.

[6] Deb K, Jain P, Gupta NK, Maji HK. Multi objective placement of electronic components using evolutionary algorithms. IEEE Trans Components Packaging Technol 2004; 27(3):480-92.

[7] Tan KC, Lee TH, Khor EF. Evolutionary algorithms with dynamic population size and local exploration for multi objective optimization. IEEE Trans Evol Comput 2001; 5(6):565-88.

[8] Van Veldhuizen DA, Zydallis JB, Lamont GB. Considerations in engineering parallel multi objective evolutionary algorithms. IEEE Trans Evol Comput 2003; 7(2):144-73.

[9] Marseguerra M, Zio E, Podofillini L. Optimal reliability/ availability of uncertain systems via multi-objective genetic algorithms. IEEE Trans Reliab 2004; 53(3):424-34.

[10] Wilson LA, Moore MD, Picarazzi JP, Miquel SDS. Parallel genetic algorithm for search and constrained multi-objective optimization. In: Proceedings of the 18th international parallel and distributed processing symposium, 26-30 April, 2004. Santa Fe, NM, USA: IEEE Computer Society; 2004.

[11] Deb K, Goel T. A hybrid multi-objective evolutionary approach to engineering shape design. In: Evolutionary multi-criterion optimization. Proceedings of the first international conference, EMO 2001, 7-9 March, 2001. Zurich, Switzerland: Springer; 2001. 